\title{
Calidez, competencia, moralidad y nacionalismo ideal como dimensiones autoestereotípicas del autoconcepto nacional en seis países de Latinoamérica
}

\author{
Warmth, Competence, Morality and Ideal Mationalism as Self-Stereotypical \\ Dimensions of the Mational Self-Concept in Six Latin American Countries \\ Sociabilidade, competência, moralidade e nacionalismo ideal como dimensões \\ auto-estereotípicas do autoconceito nacional em seis países da fmérica Latina
}

\author{
Agustín Espinosa ${ }^{*}$, Yorelis Acosta**, Juan Valencia ${ }^{* * *}$, Angela Vera ${ }^{* * * *}$, \\ Alessandro Soares da Silva ${ }^{* * * * *}$, Juan Carlos Romero ${ }^{* * *}$, Maite Beramendi ${ }^{* * * * * *}$ \\ ${ }^{*}$ Pontificia Universidad Católica del Perú/Universidade de São Paulo, Brasil. ${ }^{* *}$ Universidad Central \\ de Venezuela/Universidad Católica Andrés Bello, Venezuela. ${ }^{* * *}$ Universidad de Tarapacá, Chile. \\ ${ }^{* * * * *}$ Pontificia Universidad Católica del Perú. ${ }^{* * * * *}$ Universidade de São Paulo, Brasil. \\ ${ }^{* * * * * *}$ Universidad de Buenos Aires, Argentina/Conicet, Argentina.
}

Doi: http://dx.doi.org/10.12804/ap134.2.2016.12

\section{Resumen}

El presente estudio busca describir y analizar los contenidos autoestereotípicos relacionados con la autoestima colectiva y el grado de identificación nacional en 6 naciones de América Latina. Para tal fin, se desarrolló un estudio por encuestas en Argentina, Brasil, Chile, Colombia, Perú y Venezuela $(n=1573)$. Los resultados evidencian la presencia de cuatro dimensiones autoestereotípicas denominadas: calidez, competencia, moralidad y nacionalismo ideal. Las expresiones positivas de estas dimensiones se relacionan significativamente con la autoestima colectiva y el grado de identificación con el país. Sin embargo, las magnitudes de estas relaciones difieren según las muestras por país. De manera general, las dimensiones estereotípicas más reconocidas como

* Agustín Espinosa, profesor asociado del Departamento de Psicología de la Pontificia Universidad Católica del Perú, coordinador del Grupo de Psicología Política (GPP-PUCP), professor pesquisador visitante en la Escola de Artes, Ciência e Humanidades $(\mathrm{EACH})$ de la Universidade de São Paulo, miembro del Grupo de Estudos e Pesquisa em Psicologia Política, Políticas Públicas e Multiculturalismo de la USP (GEPSIPOLIM); ** Yorelis Acosta, profesora de la Facultad de Ciencia Política de la Universidad Central de Venezuela y profesora de Psicología Social en la Universidad Católica Andrés Bello; ***Juan Valencia, estudiante de la Escuela de Psicología de la Universidad de Tarapacá, miembro del Grupo de Psicología Política de la misma universidad; ***Juan Carlos Romero, profesor de la Escuela de Psicología de la Universidad de Tarapacá, Chile; ****Angela Vera, profesora del Departamento de Psicología de la Pontificia Universidad Católica del Perú; *****Alessandro Soares da Silva, profesor doctor de la Escola de Artes, Ciências e Humanidades de la Universidade de São Paulo (EACH-USP), coordinador del Grupo de Estudos e Pesquisa em Psicologia Política, Políticas Públicas e Multiculturalismo (GEPSIPOLIM); ******Maite Beramendi, posdoctoranda del Conicet, asistente de docencia en la Facultad de Psicología de la Universidad de Buenos Aires, Argentina.

La correspondencia relacionada con este artículo debe ser dirigida a Agustín Espinosa, Departamento Académico de Psicología, Pontificia Universidad Católica del Perú, Avenida Universitaria, 1801, Lima-32, Perú. Correo electrónico: agustin.espinosa@pucp.pe

Cómo citar este artículo: Espinosa, A., Acosta, Y., Valencia, J., Vera, A., Soares da Silva, A., Romero, J. C. \& Beramendi, M. (2016). Calidez, competencia, moralidad y nacionalismo ideal como dimensiones autoestereotípicas del autoconcepto nacional en seis países de Latinoamérica. Avances en Psicología Latinoamericana, 34(2), 395-413. doi: http://dx.doi.org/10.12804/apl34.2.2016.12 
representativas de los endogrupos nacionales fueron las de calidez y competencia; fueron menos reconocidas como representativas de estos grupos, las dimensiones de moralidad y nacionalismo ideal. No obstante, en un modelo general de las influencias de estos autoestereotipos en la autoestima colectiva y el grado de identificación, se aprecia que la calidez — aun cuando es la dimensión percibida como más representativa de los autoestereotipos nacionales - ejerce una influencia muy débil en la autoestima colectiva y no influye en el grado de identificación con el endogrupo nacional.

Palabras clave: autoestereotipos nacionales; calidez; competencia; identidad nacional; moralidad; nacionalismo ideal.

\section{fibstract}

The aim of this study is to describe and analyze self-stereotypical contents related to collective self-esteem and degree of national identification in six countries of Latin-America. To accomplish this aim, a survey study was developed in Argentina, Brazil, Chile, Colombia, Peru and Venezuela ( $n=1573)$. Results have shown four self-stereotypical dimensions: warmth, competence, morality and ideal nationalism. Positive expressions of these dimensions were significantly related to collective self-esteem and degree of national identification. Nevertheless, size effects of these relationships are different by country. In general, stereotypical dimensions perceived as more representative of the evaluated national in-groups were those related to warmth and competence; meanwhile, agreement with morality and ideal nationalism were considered less representative of them. Finally, a general model analyzing influences of self-stereotypical contents in collective self-esteem and degree of national identification showed that, even though warmth was considered the most representative dimension in the definition of the national in-group, it has a weak influence in collective self-esteem and it has no influence on the degree of national identification. Keywords: national self-stereotypes; warmth; competence; national identity; morality; ideal nationalism.

\section{Resumo}

O presente estudo busca descrever e analisar os conteúdos dos auto-estereótipos relacionados com a autoestima coletiva e o grau de identificação nacional em 6 nações da América Latina. Para tal finalidade, foi desenvolvido um estudo através da aplicação de questionários nos seguintes países: Argentina, Brasil, Chile, Colômbia, Peru e Venezuela ( $\mathrm{n}=1573)$. Os resultados mostram a presença de quatro dimensões auto-estereotípicas denominadas: sociabilidade, competência, moralidade e nacionalismo ideal. As expressões positivas destas dimensões relacionam-se significativamente com a autoestima coletiva e o grau de identificação com o país. Porém, as magnitudes destas relações diferem segundo as amostras por país. De modo geral, as dimensões estereotípicas mais reconhecidas como representativas dos ingroups nacionais foram as de sociabilidade e competência, enquanto as menos reconhecidas como representativas destes grupos, foram as dimensões de moralidade e nacionalismo ideal. Contudo, em um modelo geral das influências destes auto-estereótipos na autoestima coletiva e no grau de identificação, observa-se que a sociabilidade, mesmo sendo a dimensão percebida como a mais representativa dos auto-estereótipos nacionais, exerce uma influência muito débil na autoestima coletiva e não influi no nível de identificação com o ingroup nacional.

Palavras-chave: auto-estereótipos nacionais; competência; identidade nacional; moralidade; nacionalismo ideal; sociabilidade.

El construir la identificación con una nación tiene su base en elementos como (1) un nombre propio que define a la comunidad nacional, (2) un territorio común compartido, (3) una cultura compartida y (4) una memoria histórica de comunalidad genealógica (Herranz \& Basabe, 1999; Miles, 2000; Salazar, 1996). Adicionalmente, los elementos descritos se desprenderán de la presencia de un Estado-nación que actúe como categoría social (Béjar \& Capello, 1986; Pérez, 1999; Salazar, 1996; Salazar \& Salazar, 1998). En este escenario, 
los intentos por describir las identidades colectivas vinculadas al concepto de nación en América Latina comprenden una tarea compleja, por la diversidad social, étnica y cultural que los países de la región presentan. Así, las identidades nacionales se constituyen sobre la referencia de símbolos y abstracciones sociopolíticas y culturales hegemónicos, adheridos a espacios geográficos de gran envergadura (e.g. Carteri, 2008; Espinosa, 2011, 2003; Vives, 1994).

La psicología social ha propuesto que la identidad permite a las personas configurar su imagen a partir de un conjunto de atributos y características representativas de sí mismas, como el sexo, la etnicidad, la edad, el estatus social e incluso la nacionalidad (Miller, 1983; Owens, 2006). La identidad como constructo psicológico se define entonces como la conceptualización, en distintos niveles de representación, que una persona posee sobre sí misma. Esta representación debe entenderse como una experiencia psicológica subjetiva, antes que comprenderla como una esencia objetiva (Vignoles, Regalia, Manzi, Golledge \& Scabini, 2006).

Un subtipo específico de proceso identitario es la identidad social o colectiva, definida por Tajfel (1984) como "aquella parte del autoconcepto de un individuo que deriva del conocimiento de su pertenencia a un grupo social junto con el significado valorativo y emocional asociado a dicha pertenencia” (p. 292). La teoría de la identidad social (TIS) retoma la idea de la identidad como una experiencia subjetiva y propone que la pertenencia a un grupo es un estado psicológico (Hogg \& Abrams, 1988; Lorenzi-Cioldi \& Döise, 1990; Tajfel, 1982). En ese sentido, la identidad social connota una definición evaluativa del autoconcepto en términos de los atributos que definen al grupo, y será vista como un proceso psicológico que funciona como puente para comprender las relaciones entre diversos fenómenos sociales, la cognición social y el comportamiento individual (Hogg \& Abrams, 1988; Hogg \& Ridgeway, 2003; Tajfel, 1982).

Diversos autores sugieren que el estudio de la identidad nacional se enmarca en la definición de identidad social propuesta desde la TIS (Espinosa, 2011; Nigbur \& Cinnirella, 2007; Salazar, 1996; Smith, Giannini, Helkama, Maczynski \& Stumpf, 2005), y desde esta teoría resulta fundamental la idea de que los individuos tienen la necesidad de alcanzar y mantener una autorrepresentación positiva, como forma de asegurar la autoestima colectiva (Bar-Tal, 1996; Espinosa, 2011). De manera específica, la autoestima colectiva es uno de los componentes centrales de la identidad social y se define como la actitud de un individuo hacia las categorías y grupos sociales a los que pertenece (Luhtanen \& Crocker, 1992).

Al respecto, algunos estudios evidencian que las personas que más se identifican con una categoría nacional reportan una actitud más favorable hacia esta (Abrams \& Hogg, 1988) y hacen hincapié en representarla con más autoestereotipos positivos que describirían la "esencia" del endogrupo nacional (Smith et al., 2005; Keillor, Tomas \& Hult, 1999). Asimismo, la identificación con una categoría nacional estará vinculada al recuerdo de hechos positivos sobre el endogrupo y la omisión selectiva de eventos negativos o dramáticos, a fin de buscar mantener una imagen positiva de la nación y sus miembros (Herranz \& Basabe, 1999; Marques, Páez, Valencia \& Vincze, 2006; Páez, Marques, Valencia \& Vincze, 2006; Rottenbacher \& Espinosa, 2010).

La sistematización de la información sobre el endogrupo opera a modo de creencias grupales, $y$ la condición necesaria para que una creencia sea grupal es que tiene que estar consensuada entre aquellas personas que comparten una categoría social (Bar-Tal, 1996). Los estereotipos sociales son estructuras cognitivas que simplifican las relaciones sociales determinando la pertenencia de un individuo a un grupo o categoría social, a partir de un conjunto de rasgos perceptuales predominantes (Stangor, 2009). Los estereotipos son contextualizados y transmitidos mediante la socialización y se llegan a constituir como creencias sociales (Stangor, 2009). 
Estudios recientes sobre contenidos estereotípicos sugieren que están conformados por dos dimensiones: una de calidez y otra de competencia (Fiske, Cuddy \& Glick, 2003; Fiske, Cuddy, Glick $\& \mathrm{Xu}, 2002)$. Si bien esta aproximación se basa en las representaciones sobre el exogrupo, en el presente trabajo se propone que esta podría aplicar también para el análisis de las representaciones estereotípicas sobre el endogrupo. De manera específica, la calidez se relaciona con la percepción de los miembros de un grupo social como tolerantes, cálidos, buenos y sinceros; por otra parte, la competencia involucra características atribuidas a las capacidades de acción de los miembros de un grupo en aspectos como ser competentes, inteligentes, confiables y competitivos (Fiske et al., 2003; Fiske et al., 2002). Distintas respuestas afectivas hacia un grupo social derivarán de las combinatorias de estas dimensiones. Así, un individuo puede sentir admiración por los miembros de un grupo cálido y competente; envidia hacia un grupo competente, no cálido; tristeza por los cálidos incompetentes, $\mathrm{y}$ desprecio por los incompetentes, no cálidos $(\mathrm{Cu}-$ ddy, Fiske \& Glick, 2007; Fiske et al., 2003). De estas representaciones estereotípicas derivan las percepciones sobre estatus y competencia atribuidas a distintos grupos sociales (Cuddy et al., 2007; Fiske et al., 2003; Fiske et al., 2002).

Lo anterior es interesante, porque una buena parte de los estudios sobre identidad nacional en América Latina se han orientado a describir y analizar los contenidos autoestereotípicos relacionados con las categorías nacionales de los países de la región (Salazar \& Salazar, 1998). En esta línea de investigación, se aprecia una producción interesante de documentos durante los años noventa (e. $g$., Banchs, Cadenas, Domínguez \& Montero, 1993; D’Adamo \& García Beaudoux, 1995; Montero, 1996a; Salazar \& Salazar, 1998; Salgado, 1999) y una consolidación de estos trabajos en décadas posteriores (e. g., Beramendi \& Zubieta, 2013; Espinosa, 2003, 2011; Espinosa \& Tapia, 2011;
Monsegur, Espinosa \& Beramendi, 2014; Pérez de León, 2007; Roselli, 2000).

Por una parte, los estudios de la década de los noventa daban cuenta de representaciones autoestereotípicas predominantemente negativas, lo que sugería la presencia de identidades sociales potencialmente negativas en América Latina (D'Adamo \& García Beaudoux, 1995; Montero, 1996a, 1996b; Morales \& Páez, 1996). Estos resultados mostraban cierta tendencia a la desvalorización de los endogrupos nacionales con representaciones de baja competencia y baja moralidad (Roselli, 2000; Salazar \& Salazar, 1998); mientras se mostraba cierto favoritismo hacia exogrupos nacionales percibidos como de mayor desarrollo, instrumentalismo y estatus, en un fenómeno denominado altercentrismo (Montero, 1992, 1996b). Los estudios de décadas posteriores evidencian cierta persistencia de contenidos autoestereotípicos negativos asociados a las categorías nacionales en América Latina. Sin embargo, en estas investigaciones emergen con mayor claridad algunos contenidos autoestereotípicos positivos relacionados con la calidez y el incremento de la competencia, lo que produce representaciones identitarias ambivalentes en respuesta a las representaciones marcadamente negativas de la década anterior (e. g., Beramendi \& Zubieta, 2013; Espinosa, 2011; Monsegur et al., 2014; Roselli, 2000).

La evolución de las representaciones identitarias en América Latina corrobora la idea de que la construcción de la identidad se encuentra alineada con la percepción sobre el contexto en que esta se desarrolla (e.g., Beramendi, 2014; Beramendi \& Zubieta, 2013; Espinosa, 2011). Así, las identificaciones sociales negativas de la década de los noventa son una resultante de los escenarios de crisis política, social y económica que muchos países de la región atravesaron durante los años ochenta y noventa (e.g., Montero, 1996a; Salazar \& Salazar, 1998). Mientras que las representaciones actuales suponen una reconfiguración de algunos contenidos negativos, que ahora se tornaron positivos, y el reconocimiento de otros tantos contenidos positivos, 
aunque todavía persiste cierta negatividad (e.g., Espinosa, 2011; Monsegur et al., 2014).

Sobre lo revisado, el objetivo del presente estudio es intentar describir y analizar cómo se estructuran los contenidos autoestereotípicos en muestras de Argentina, Brasil, Chile, Colombia, Perú y Venezuela, y cómo estos contenidos autoestereotípicos se relacionan con el grado de identificación y la valoración de la pertenencia a estas naciones.

\section{Método}

\section{Participantes}

Participaron en el estudio 1573 personas de seis países de América del Sur (Argentina, Brasil, Chile, Colombia, Perú y Venezuela), del total de la muestra el $51.6 \%$ fueron hombres. Las edades fluctuaron entre los 17 y los 81 años $(M=28.54$; $D E=12.20)$. La mayoría de los participantes en el estudio se autocategorizó como de clase media (55.5\%), un segundo grupo se autocategorizó como de clase media-alta (27.2\%) y en un tercer grupo están aquellos que se presentaron como de clase media-baja (13\%). Finalmente, están los grupos que se autocategorizaron como de clase alta $(2.4 \%)$ y clase baja (1.9\%).

Tabla 1

Características demográficas de la muestra por país

\begin{tabular}{|c|c|c|c|c|c|c|c|c|}
\hline & & $\begin{array}{c}\text { Argentina } \\
n=157\end{array}$ & $\begin{array}{c}\text { Brasil } \\
n=156\end{array}$ & $\begin{array}{c}\text { Chile } \\
n=196\end{array}$ & $\begin{array}{c}\text { Colombia } \\
n=203\end{array}$ & $\begin{array}{c}\text { Perú } \\
n=479\end{array}$ & $\begin{array}{c}\text { Venezuela } \\
n=382\end{array}$ & $\begin{array}{c}\text { Total } \\
n=1573\end{array}$ \\
\hline \multirow{2}{*}{ Sexo } & Hombre (\%) & 24.8 & 58.7 & 49 & 35.6 & 45.4 & 76.4 & 51.6 \\
\hline & Mujer (\%) & 75.2 & 41.3 & 51 & 64.4 & 54.6 & 23.6 & 48.4 \\
\hline Edad & $\begin{array}{l}M \\
(D E)\end{array}$ & $\begin{array}{l}24.64 \\
(9.53)\end{array}$ & $\begin{array}{l}24.31 \\
(9.83)\end{array}$ & $\begin{array}{c}29.42 \\
(11.31)\end{array}$ & $\begin{array}{c}33 \\
(12.47)\end{array}$ & $\begin{array}{c}30.45 \\
(11.62)\end{array}$ & $\begin{array}{c}26.73 \\
(13.69)\end{array}$ & $\begin{array}{c}28.54 \\
(12.20)\end{array}$ \\
\hline \multirow{5}{*}{$\begin{array}{l}\text { Clase social } \\
\text { autopercibida }\end{array}$} & Alta $(\%)$ & 0.0 & .0 & 1.5 & 1.0 & 5.2 & 1.8 & 2.4 \\
\hline & Media-alta (\%) & 15.9 & 10.5 & 18.4 & 33.6 & 36.3 & 28.4 & 27.2 \\
\hline & Media (\%) & 72.0 & 55.9 & 48.5 & 57.6 & 49.3 & 58.7 & 55.5 \\
\hline & Media-baja (\%) & 10.8 & 28.3 & 25.5 & 7.4 & 8.8 & 9.7 & 13.0 \\
\hline & Baja (\%) & 1.3 & 5.3 & 6.1 & 5.0 & .4 & 1.3 & 1.9 \\
\hline
\end{tabular}

Adicionalmente a la descripción general de la muestra del estudio, es importante mencionar que algunas características demográficas de los participantes difieren entre países. En ese sentido, la tabla 1 presenta de manera específica las características de cada muestra por país.

\section{Medidas e instrumentos de recolección de información}

\section{Subescala de Autoestima Colectiva Privada} (Luhtanen \& Crocker, 1992). La subescala posee cuatro ítems que evalúan la relación afectiva de los participantes respecto a su identificación con su país. Se plantearon preguntas como: "En general, me siento afortunado por ser categoría social" o "Generalmente, siento que ser categoría social no merece la pena". Las respuestas van del 1 al 5 en una escala tipo Likert, donde 1 es "Totalmente en desacuerdo" y 5 es "Totalmente de acuerdo". En el caso de Argentina, Chile, Colombia, Perú y Venezuela, se utilizó la versión española de la escala con adaptaciones a las categorías nacionales correspondientes a cada país. En el caso de Brasil, se realizó una traducción cruzada de la referida versión española. La consistencia interna de la subescala para el total de la muestra fue muy buena 
( $\alpha=.84)$. La confiabilidad por países estuvo entre aceptable y muy buena: Argentina $(\alpha=.79)$, Brasil $(\alpha=.81)$, Chile $(\alpha=.87)$, Colombia $(\alpha=.86)$, Perú $(\alpha=.84)$ y Venezuela $(\alpha=.82)$.

Grado de identificación con el país (Espinosa, 2011). Se utilizó un ítem que mide el grado de identificación que posee un individuo con los miembros de su país. Se planteó la siguiente pregunta: ¿cuál es su grado de identificación con categoría nacional? La pregunta fue adaptada a cada categoría nacional. La escala de respuestas va del 1 al 5, donde 1 es "Nada" y 5 es "Total".

Escala de AutoEstereotipos Nacionales (Espinosa, 2011). La escala consta de 22 ítems, que comprenden una lista de 22 adjetivos positivos y sus respectivos antónimos que caracterizan que tanto esos atributos son representativos del endogrupo nacional. La versión utilizada de la escala es la versión desarrollada para Argentina (Espinosa \& Beramendi, 2012), sobre la cual se desarrollaron nuevas adaptaciones para actualizar semánticamente algunos adjetivos del cuestionario a las realidades de los 6 países participantes en el estudio. Cabe resaltar que para el caso brasileño, la escala fue sometida a un proceso de traducción cruzada al idioma portugués. La escala de respuestas por ítem está estructurada como un diferencial semántico de 7 puntos. Así, por ejemplo, los participantes debían marcar el número que más se acercaba al adjetivo que creyeran era distintivo de las personas de su país. Entonces, en la dupla de adjetivos solidarios-egoístas: 1 sería el acuerdo con que los miembros del endogrupo nacional son muy solidarios; 4, el acuerdo con que los miembros del endogrupo no son ni solidarios, ni egoístas, y 7, el acuerdo con que los miembros del endogrupo son muy egoístas. Los análisis sobre las dimensiones autoestereotípicas derivadas de la escala y su consistencia interna se vinculan a los objetivos del presente estudio y serán reportados como resultados en el acápite correspondiente. Se invirtieron los valores de la escala por ítem para conseguir que la mayor puntuación en el atributo corresponda a la valoración positiva de este.

\section{Procedimiento}

El levantamiento de información se realizó entre septiembre de 2013 y mayo de 2014. La recolección de datos se desarrolló de dos maneras: una presencial en contextos educativos, donde se aplicaba el cuestionario a estudiantes universitarios, y una virtual, a través de la plataforma google.docs, que permitía tener acceso a población general. El uso específico de una versión virtual es reconocida como un método equivalente, e incluso con algunas ventajas sobre las aplicaciones presenciales (Lyons, Cude, Lawrence \& Gutter, 2005). Se argumenta que entre las ventajas identificadas en el uso de cuestionarios virtuales están el incremento en la diversidad de la muestra, una mayor rapidez y una reducción de costos en el proceso de recolección de datos (cf. Weber \& Bradley, 2006). Asimismo, disminuye la deseabilidad social en la respuesta y reduce errores vinculados al proceso tabulación de datos (cf. Lyons et al., 2005). Estos beneficios han sido corroborados en el presente estudio.

La aplicación de los cuestionarios, en ambas versiones, vino acompañada de un consentimiento informado que daba cuenta del objetivo general del estudio, el carácter voluntario de la participación, el respeto por la confidencialidad de las respuestas, la salvaguarda del anonimato de los participantes y el uso de la información con fines estrictamente académicos.

La información obtenida se sometió a un procesamiento estadístico a partir de técnicas descriptivas, correlacionales, inferenciales y multivariadas pertinentes para los objetivos del estudio.

\section{Resultados}

\section{Dimensiones autoestereotípicas}

Para identificar la estructura de los contenidos autoestereotípicos, los 22 ítems de la escala de autoestereotipos nacionales se sometieron a un análisis factorial exploratorio con una selección 
aleatoria de aproximadamente un 50\% de los casos de la muestra total $(n=814)$. En el presente análisis se utilizó el método de componentes principales y una rotación ortogonal de tipo varimax.

Los resultados del análisis factorial exploratorio evidencian la adecuación de la muestra a una clara estructura $(K M O=.930)$ de 4 dimensiones que explican una varianza del $57.23 \%$. La tabla 2 muestra las 4 dimensiones encontradas, los ítems que las conforman con sus respectivas cargas factoriales; así como la varianza explicada por factor y su consistencia interna.

En un segundo momento se realizó un análisis factorial confirmatorio con la otra mitad del total de la muestra $(n=759)$. Se evaluó la estructura de 4 factores con el método de máxima verosimilitud

Tabla 2

Análisis factorial exploratorio de la Escala de Autoestereotipos Nacionales

\begin{tabular}{|c|c|c|c|c|}
\hline Ítems & $\begin{array}{l}\text { Dimensión 1: } \\
\text { moralidad }\end{array}$ & $\begin{array}{l}\text { Dimensión 2: } \\
\text { calidez }\end{array}$ & $\begin{array}{l}\text { Dimensión 3: } \\
\text { competencia }\end{array}$ & $\begin{array}{c}\text { Dimensión 4: } \\
\text { nacionalismo ideal }\end{array}$ \\
\hline Honestos-deshonestos & .82 & & & \\
\hline Confiables-no confiables & .76 & & & \\
\hline Sinceros-mentirosos & .74 & & & \\
\hline Honrados-corruptos & .74 & & & \\
\hline Considerados/respetuosos-prepotentes/irrespetuosos & .64 & & & \\
\hline Solidarios-egoístas & .61 & & & \\
\hline Cultos-ignorantes & .53 & & & \\
\hline Expresivos/comunicativos-inexpresivos/no comunicativos & & .78 & & \\
\hline Sociables-no sociables & & .77 & & \\
\hline Alegres-tristes/melancólicos & & .73 & & \\
\hline Simpáticos-antipáticos & & .73 & & \\
\hline Vivos/piolas/astutos-sonsos/giles & & .59 & & \\
\hline Adaptativos/flexibles-no adaptativos/rígidos & & .58 & & \\
\hline Emprendedor/proactivos-no emprendedores/no activos & & & .74 & \\
\hline Trabajadores-ociosos/vagos & & & .67 & \\
\hline Creativos-no creativos & & & .63 & \\
\hline Capaces-incapaces & & & .63 & \\
\hline Inteligentes-tontos & & & .56 & \\
\hline Exitosos-fracasados & & & .53 & \\
\hline Cuestionadores-sumisos & & & & .75 \\
\hline Desarrollados-subdesarrollados & & & & .56 \\
\hline Patriotas-no patriotas & & & & .51 \\
\hline Varianza explicada (\%) & 34.36 & 12.06 & 6.07 & 4.79 \\
\hline Consistencia interna & .88 & .83 & .82 & .51 \\
\hline
\end{tabular}




\section{Agustín Espinosa et al.}

y se obtuvieron niveles de ajuste y error aceptables. $\chi^{2} / g l=4.92 ; R M R=.131 ; G F I=.888 ; C F I=.891$; $R M S E A=.07$. La tabla 3 muestra las 4 dimensiones evaluadas, los ítems que las conforman con sus respectivos betas estandarizados y la consistencia interna por dimensión.

Sobre este punto, los indicadores de consistencia interna de las dimensiones utilizando los 1573 casos de la muestra fueron los siguientes: moralidad, $\alpha=.87$; calidez, $\alpha=.84$; competencia, $\alpha=.82$; y nacionalismo ideal, $\alpha=.50$. Los coeficientes de consistencia interna obtenidos resultan aceptables para los posteriores análisis, considerando el metanálisis de Mezulis, Abramson, Hyde y Hankin (2004), donde los autores encuentran que en las escalas que incorporan algún criterio de autopresentación existe cierta estabilidad y sesgo positivo en las respuestas, lo que suele reducir las

Tabla 3

Análisis factorial confirmatorio de la Escala de Autoestereotipos Nacionales

\begin{tabular}{|c|c|c|c|c|}
\hline Ítems & $\begin{array}{l}\text { Dimensión } \\
\text { 1: moralidad }\end{array}$ & $\begin{array}{l}\text { Dimensión } \\
\text { 2: calidez }\end{array}$ & $\begin{array}{l}\text { Dimensión 3: } \\
\text { competencia }\end{array}$ & $\begin{array}{c}\text { Dimensión 4: } \\
\text { nacionalismo idea }\end{array}$ \\
\hline Honestos-deshonestos & .73 & & & \\
\hline Confiables-no confiables & .75 & & & \\
\hline Sinceros-mentirosos & .80 & & & \\
\hline Honrados-corruptos & .74 & & & \\
\hline Considerados/respetuosos-prepotentes/irrespetuosos & .67 & & & \\
\hline Solidarios-egoístas & .59 & & & \\
\hline Cultos-ignorantes & .67 & & & \\
\hline Expresivos/comunicativos-inexpresivos/no comunicativos & & .76 & & \\
\hline Sociables-no sociables & & .78 & & \\
\hline Alegres-tristes/melancólicos & & .76 & & \\
\hline Simpáticos-antipáticos & & .77 & & \\
\hline Vivos/piolas/astutos-sonsos/giles & & .59 & & \\
\hline Adaptativos/flexibles-no adaptativos/rígidos & & .55 & & \\
\hline Emprendedor/proactivos-no emprendedores/no activos & & & .66 & \\
\hline Trabajadores-ociosos/vagos & & & .59 & \\
\hline Creativos-no creativos & & & .59 & \\
\hline Capaces-incapaces & & & .77 & \\
\hline Inteligentes-tontos & & & .76 & \\
\hline Exitosos-fracasados & & & .63 & \\
\hline Cuestionadores-sumisos & & & & .41 \\
\hline Desarrollados-subdesarrollados & & & & .65 \\
\hline Patriotas-no patriotas & & & & .38 \\
\hline Consistencia interna & .87 & .85 & .82 & .48 \\
\hline
\end{tabular}


confiabilidades, por lo que resulta apropiado trabajar hasta con coeficientes de .50 .

\section{Descriptivos del grado de identificación, autoestima colectiva y dimensiones autoestereotípicas}

Los análisis que se presentan a continuación fueron realizados con el total de los casos de la muestra $(n=1573)$. Con relación al grado de identificación y a la autoestima colectiva, se tiene que en una escala del 1 al 5, el nivel general de identificación con el país se encuentra por encima del punto medio de dicha escala $(M=3.87 ; D E=1.05)$. Un resultado similar se aprecia en el caso de la autoestima colectiva $(M=3.98 ; D E=.94)$.

Los análisis de varianza sugieren diferencias significativas en los niveles de identificación, $F(5,1567)=18.37, p<.001$ y en la autoestima colectiva, $F(5,1567)=27.57, p<.001$ por países. $\mathrm{La}$ tabla 4 presenta las medias y desviaciones estándar de las referidas variables

Análisis post hoc de Bonferroni muestran diferencias específicas para el grado de identificación y la autoestima colectiva entre los países. De manera específica, en el caso del grado de identificación, se aprecian dos grupos claramente diferenciados. Así, Colombia, Perú y Venezuela, sistemáticamente, presentan identificaciones significativamente más altas que Argentina, Brasil y Chile $\left(p_{s}<.001\right)$. En el caso de la autoestima colectiva, Colombia, con la puntuación más alta, se diferencia de todos los países, con excepción de Perú $\left(p_{s}<.001\right)$. Luego vienen Perú, Argentina y Venezuela, que no se diferencian entre sí; mas sí se diferencian de Chile y Brasil $\left(p_{s}<.05\right)$ que son los países con puntuaciones más bajas en esta característica.

Con relación a las cuatro dimensiones autoestereotípicas identificadas por los análisis factoriales, en una escala del 1 al 7, donde la proximidad al valor 1 significa un mayor acuerdo con la connotación negativa de la dimensión y el 7 un mayor acuerdo con la dimensión positiva, se aprecia que el mayor consenso en los participantes es que los miembros de sus endogrupos nacionales son caracterizados por la calidez $(M=5.51 ; D E=1.30)$ y la competencia $(M=5.11 ; D E=.99)$. Más distantes aparecen los atributos de moralidad $(M=3.94 ; D E=1.15)$ y nacionalismo ideal $(M=3.91 ; D E=1.09)$.

Análisis descriptivos muestran que el orden general de atributos descrito no se reproduce de la misma manera por país. Así, la calidez es el atributo más consensual en Argentina, Brasil, Colombia, Perú y Venezuela, mas no en Chile, donde ocupa el segundo lugar. La competencia es el segundo atributo más positivo en Argentina, Brasil, Colombia, Perú y Venezuela; pero resulta primero en Chile. El nacionalismo ideal es el atributo que ocupa el tercer lugar en Argentina, Chile y Perú, y relega la moralidad al último lugar en estos países. En Brasil, Colombia y Venezuela se aprecia la tendencia inversa.

Adicionalmente, al margen de las diferencias en el orden de los atributos autoestereotípicos por país, diversos análisis de varianza muestran diferencias específicas en la valoración de la calidez, $F(5,1567)$ $=101.61, p<.001 ;$ competencia, $F(5,1567)=$ $35.31, p<.001$; moralidad, $F(5,1567)=12.37$,

Tabla 4

Descriptivos de grado de identificación y autoestima colectiva por país

\begin{tabular}{lcrrrrrrr}
\hline & Argentina & Brasil & Chile & Colombia & Perú & Venezuela & Total \\
\hline \multirow{2}{*}{ Grado de identificación } & 3.62 & 3.51 & 3.45 & 4.13 & 4.02 & 4.01 & 3.87 \\
& $(.87)$ & $(1.11)$ & $(1.22)$ & $(1.00)$ & $(.93)$ & $(1.10)$ & $(1.05)$ \\
\hline \multirow{2}{*}{ Autoestima colectiva } & 3.97 & 3.62 & 3.46 & 4.31 & 4.12 & 4.01 & 3.98 \\
& $(.80)$ & $(.96)$ & $(1.07)$ & $(.87)$ & $(.80)$ & $(.95)$ & $(.94)$ \\
\hline
\end{tabular}


$p<.001$; y el nacionalismo ideal, $F(5,1567)=$ $22.96, p<.001$. La tabla 5 las medias y desviaciones estándar de las referidas variables.

Análisis post hoc de Bonferroni muestran diferencias específicas con relación al autoestereotipo de calidez entre los países. Así, Venezuela, que presenta la mayor puntuación en este atributo, no se diferencia de Colombia, pero sí del resto de países $\left(p_{s}<.001\right)$. Colombia, en segundo lugar, no se diferencia de Brasil, pero sí del resto de países $\left(p_{s}<.001\right)$. Por su parte, Brasil, en tercer lugar, se diferencia de Argentina $(p<.01)$, Perú y Chile $\left(p_{s}<\right.$ $.001)$. Argentina y Perú, en cuarto y quinto lugares, no se diferencian entre sí, pero sí de Chile que tiene la puntuación más baja en este atributo $(p<.001)$.

Sobre el autoestereotipo de competencia, los análisis de Bonferroni muestran a Colombia con la puntuación más elevada en este contenido y se diferencia claramente de todos los países $\left(p_{s}<.001\right)$. Luego, en segundo y tercer lugares, vienen Perú y Venezuela, que no se diferencian entre sí, pero sí lo hacen de Argentina $(p<.01)$, Brasil y Chile de manera significativa $\left(p_{s}<.001\right)$. Finalmente, Argentina, Brasil y Chile no logran diferenciarse entre sí, según sus puntuaciones.

En cuanto al autoestereotipo de moralidad, los análisis de Bonferroni muestran que Colombia, con la puntuación más elevada, se diferencia significativamente de Venezuela $(p<.01)$ y el resto de países $\left(p_{s}<.001\right)$. Venezuela, en segundo lugar, no se diferencia de Argentina, mas sí de Perú y Chile $\left(p_{s}<.05\right)$ y de Brasil $\left(p_{s}<.01\right)$. Argentina, Brasil, Perú y Chile no presentan diferencias estadísticas entre sí.

Respecto al nacionalismo ideal, los análisis de Bonferroni permiten apreciar que Argentina, país que presenta la mayor puntuación en el atributo, se diferencia de todos los países $\left(p_{s}<.001\right)$ con excepción de Colombia. Por su parte, Colombia, en segundo lugar, no se diferencia de Chile; pero sí de Brasil, Perú y Venezuela $\left(p_{s}<.001\right)$. Chile, Perú y Venezuela conforman un conglomerado que no se diferencia entre sí, pero que sí se diferencia de Brasil en cada uno de los casos $\left(p_{s}<.001\right)$.

\section{Relaciones entre el grado de identificación, la autoestima colectiva y los autoestereotipos nacionales}

De acuerdo con lo esperado, se encuentran relaciones significativas con tamaños del efecto de moderados a altos (cf. Hemphill, 2003; Richard, Bond Jr. \& Stokes-Zoota, 2003) entre el grado de identificación, la autoestima colectiva y las dimensiones del autoestereotipo nacional. Así, la identificación y la autoestima colectiva presentan una relación directa, $r(1572)=.63, p<.001$. Las relaciones descritas, aunque significativas en casi todos los casos, difieren en las magnitudes del efecto según país. Así, la relación entre el grado

Tabla 5

Descriptivos de las dimensiones autoestereotípicas por país

\begin{tabular}{lcrrrrrrr}
\hline & Argentina & \multicolumn{1}{c}{ Brasil } & \multicolumn{1}{c}{ Chile } & Colombia & Perú & Venezuela & Total \\
\hline \multirow{2}{*}{ Calidez } & 5.29 & 5.68 & 4.55 & 5.93 & 5.24 & 6.13 & 5.51 \\
& $(.79)$ & $(1.04)$ & $(1.07)$ & $(.83)$ & $(.94)$ & $(.75)$ & $(1.30)$ \\
\hline \multirow{2}{*}{ Competencia } & 4.85 & 4.73 & 4.62 & 5.67 & 5.22 & 5.17 & 5.11 \\
& $(.76)$ & $(.92)$ & $(.98)$ & $(.98)$ & $(.88)$ & $(.98)$ & $(.99)$ \\
\hline \multirow{2}{*}{ Moralidad } & 3.85 & 3.63 & 3.74 & 4.36 & 3.80 & 4.03 & 3.94 \\
& $(.88)$ & $(.93)$ & $(1.13)$ & $(1.17)$ & $(1.06)$ & $(1.06)$ & $(1.15)$ \\
\hline \multirow{2}{*}{ Nacionalismo ideal } & 4.54 & 3.31 & 4.00 & 4.24 & 3.88 & 3.85 & 3.91 \\
& $(.88)$ & $(1.09)$ & $(1.07)$ & $(1.30)$ & $(1.07)$ & $(1.17)$ & $(1.09)$
\end{tabular}


de identificación y la autoestima colectiva presenta los siguientes valores por país: Argentina, $r(156)$ $=.34, p<.001$; Brasil, $r(155)=.64, p<.001$; Chile, $r(195)=.71, p<.001$; Colombia, $r(202)=.63$, $p<.001$; Perú, $r(478)=.64, p<.001$ y Venezuela, $r(381)=.58, p<.001$.

Luego, en general, el grado de identificación se asocia positivamente a la calidez, $r(1572)=.31$, $p<.001$; la competencia, $r(1572)=.46, p<.001$; la moralidad, $r(1572)=.42, p<.001$ y el nacionalismo ideal, $r(1572)=.31, p<.001$. De igual manera, la autoestima colectiva se relaciona con la calidez, $r(1572)=.32, p<.001$; la competencia, $r(1572)=.47, p<.001$; la moralidad, $r(1572)$ $=.45, p<.001 \mathrm{y}$ el nacionalismo ideal, $r(1572)$ $=.36, p<.001$. La tabla 6 resume las relaciones entre el grado de identificación y la autoestima colectiva por una parte y las dimensiones autoestereotípicas por otra parte.

De la misma manera, las relaciones entre los autoestereotipos son significativas y sugieren que a mayor contenido positivo en uno, mayor contenido en otro. Así, la calidez correlaciona positivamente con la competencia, $r(1572)=.57, p<.001$; la moralidad, $r(1572)=.40, p<.001 \mathrm{y}$ el nacionalismo ideal, $r(1572)=.24, p<.001$. La competencia se relaciona positivamente con la moralidad, $r(1572)$ $=.59 p<.001 \mathrm{y}$ el nacionalismo ideal, $r(1572)$ $=.44, p<.001$. Finalmente, la moralidad se relaciona positivamente con el nacionalismo ideal, $r(1572)=.54, p<.001$. La tabla 7 resume las relaciones que las dimensiones autoestereotípicas presentan entre sí por país.

Tabla 6

Relaciones entre el grado de identificación y la autoestima colectiva con las dimensiones autoestereotípicas por país

\begin{tabular}{lcccccc}
\hline & Argentina & Brasil & Chile & Colombia & Perú & Venezuela \\
\hline Grado de identificación-calidez & $.31^{* * *}$ & $.16^{*}$ & $.45^{* * *}$ & $.33^{* * *}$ & $.32^{* * *}$ & $.15^{* *}$ \\
Grado de identificación-competencia & $.41^{* * *}$ & $.45^{* * *}$ & $.49^{* * *}$ & $.50^{* * *}$ & $.43^{* * *}$ & $.33^{* * *}$ \\
Grado de identificación moralidad & $.32^{* * *}$ & $.56^{* * *}$ & $.43^{* * *}$ & $.50^{* * *}$ & $.40^{* * *}$ & $.35^{* * *}$ \\
Grado de identificación- nacionalismo ideal & $.16^{*}$ & $.38^{* * *}$ & $.37^{* * *}$ & $.37^{* * *}$ & $.33^{* * *}$ & $.31^{* * *}$ \\
Autoestima colectiva-calidez & $.21^{* *}$ & $.20^{*}$ & $.44^{* * *}$ & $.36^{* * *}$ & $.40^{* * *}$ & $.16^{* *}$ \\
Autoestima colectiva-competencia & $.27^{* * *}$ & $.47^{* * *}$ & $.43^{* * *}$ & $.44^{* * *}$ & $.48^{* * *}$ & $.37^{* * *}$ \\
Autoestima colectiva- moralidad & $.38^{* * *}$ & $.57^{* * *}$ & $.53^{* * *}$ & $.45^{* * *}$ & $.41^{* * *}$ & $.41^{* * *}$ \\
Autoestima colectiva-nacionalismo ideal & $.28^{* * *}$ & $.35^{* * *}$ & $.42^{* * *}$ & $.39^{* * *}$ & $.36^{* * *}$ & $.37^{* * *}$ \\
\hline
\end{tabular}

${ }^{* * *} \mathrm{p}<.001 ; * \mathrm{p}<.01 ;{ }^{*} \mathrm{p}<.05$

Tabla 7

Relaciones entre dimensiones autoestereotípicas por país

\begin{tabular}{lcccccc}
\hline & Argentina & Brasil & Chile & Colombia & Perú & Venezuela \\
\hline Calidez-competencia & $.45^{* * *}$ & $.56^{* * *}$ & $.58^{* * *}$ & $.74^{* * *}$ & $.68^{* * *}$ & $.46^{* * *}$ \\
Calidez-moralidad & $.36^{* * *}$ & $.31^{* * *}$ & $.56^{* * *}$ & $.47^{* * *}$ & $.40^{* * *}$ & $.32^{* * *}$ \\
Calidez-nacionalismo ideal & $.32^{* * *}$ & .03 & $.53^{* * *}$ & $.39^{* * *}$ & $.40^{* * *}$ & $.15^{* *}$ \\
Competencia-moralidad & $.44^{* * *}$ & $.59^{* * *}$ & $.68^{* * *}$ & $.62^{* * *}$ & $.53^{* * *}$ & $.61^{* * *}$ \\
Competencia- nacionalismo ideal & $.41^{* * *}$ & $.42^{* * *}$ & $.35^{* * *}$ & $.51^{* * *}$ & $.47^{* * *}$ & $.48^{* * *}$ \\
Moralidad-nacionalismo ideal & $.40^{* * *}$ & $.51^{* * *}$ & $.50^{* * *}$ & $.69^{* * *}$ & $.58^{* * *}$ & $.48^{* * *}$ \\
\hline
\end{tabular}

${ }^{* * *} \mathrm{p}<.001,{ }^{* *} \mathrm{p}<.01,{ }^{*} \mathrm{p}<.05$ 


\section{Segmentos de ciudadanos según las dimensiones del autoestereotipo colectivo}

Se procesó un análisis de conglomerados de K-medias para evaluar cómo las cuatro dimensiones autoestereotípicas dividían a los participantes en distintos grupos. Se obtuvieron tres grupos de ciudadanos, tal como se observa en la tabla 8 .

Tabla 8

Análisis de conglomerados de K-medias de las dimensiones del autoestereotipo nacional. Centros finales de los conglomerados

\begin{tabular}{lccc}
\hline & \multicolumn{3}{c}{ Conglomerados } \\
\cline { 2 - 4 } & Pesimistas & Optimistas & Ambivalentes \\
\hline Calidez & 4.16 & 6.13 & 5.78 \\
\hline Competencia & 4.06 & 5.94 & 5.03 \\
\hline Moralidad & 3.03 & 4.93 & 3.58 \\
\hline $\begin{array}{l}\text { Nacionalismo } \\
\text { ideal }\end{array}$ & 3.34 & 4.93 & 3.50 \\
\hline
\end{tabular}

El primer conglomerado estuvo conformado por 377 personas y muestra percepciones de calidez y competencia medias, y percepciones de moralidad y nacionalismo ideal bajas. Este conglomerado sería el conformado por aquellos que presentan la imagen más negativa de su endogrupo nacional y sería denominado como el grupo de los "pesimistas". El segundo conglomerado está conformado por 534 personas que reportan un acuerdo alto con los estereotipos de calidez y competencia, y una acuerdo medio-alto con los estereotipos de moralidad y nacionalismo ideal. Este segundo grupo presenta las percepciones más positivas del endogrupo nacional y sería denominado como el grupo de los “optimistas". El tercer grupo presenta puntuaciones altas en las dimensiones de calidez y competencia, y puntuaciones medias en moralidad y nacionalismo ideal. Este sería un grupo denominado "ambivalente".

La distribución por grupo de los participantes por país muestra diferencias significativas, $\chi^{2}(10)$
$=244.26, p<.001$. La distribución por grupo de los participantes por país se presenta en la tabla 9 .

Tabla 9

Distribución por conglomerados de participantes por pais (en porcentajes)

\begin{tabular}{lcccc}
\hline & Pesimistas & Optimistas & Ambivalentes & Total \\
\hline $\begin{array}{l}\text { Argentina } \\
(n=157)\end{array}$ & 27.4 & 35.7 & 36.9 & 100 \\
\hline $\begin{array}{l}\text { Brasil } \\
(n=156)\end{array}$ & 21.2 & 16.7 & 62.2 & 100 \\
\hline $\begin{array}{l}\text { Chile } \\
(n=196)\end{array}$ & 55.6 & 22.4 & 21.9 & 100 \\
\hline $\begin{array}{l}\text { Colombia } \\
(n=203)\end{array}$ & 9.4 & 54.2 & 26.5 & 100 \\
\hline $\begin{array}{l}\text { Perú } \\
(n=479)\end{array}$ & 29.4 & 31.9 & 38.6 & 100 \\
\hline $\begin{array}{l}\text { Venezuela } \\
(n=382)\end{array}$ & 8.4 & 38.0 & 53.7 & 100 \\
\hline $\begin{array}{l}\text { Total } \\
(n=1573)\end{array}$ & 24.0 & 33.9 & 42.1 & 100 \\
\hline
\end{tabular}

Análisis de senderos de las influencias de los autoestereotipos en la identidad nacional. Un modelo integrado

Finalmente, a pesar de las diferencias específicas por país observadas en el plano descriptivo e inferencial, se buscó analizar un modelo general para los seis países, de las influencias de los autoestereotipos nacionales en la autoestima colectiva y la identificación nacional. El planteamiento del modelo hipotético está basado conceptualmente en las propuestas de Smith et al. (2005) y Espinosa (2011), las cuales sugieren que los autoestereotipos son predictores de la valoración y del nivel de identificación con un endogrupo nacional.

Así, el primer modelo presentaba como variables exógenas las cuatro dimensiones autoestereotípicas, que - se esperaba - en un primer momento influenciaran positivamente la autoestima colectiva y en un segundo momento hicieran su parte en las cuatro dimensiones autoestereotípicas y de la 


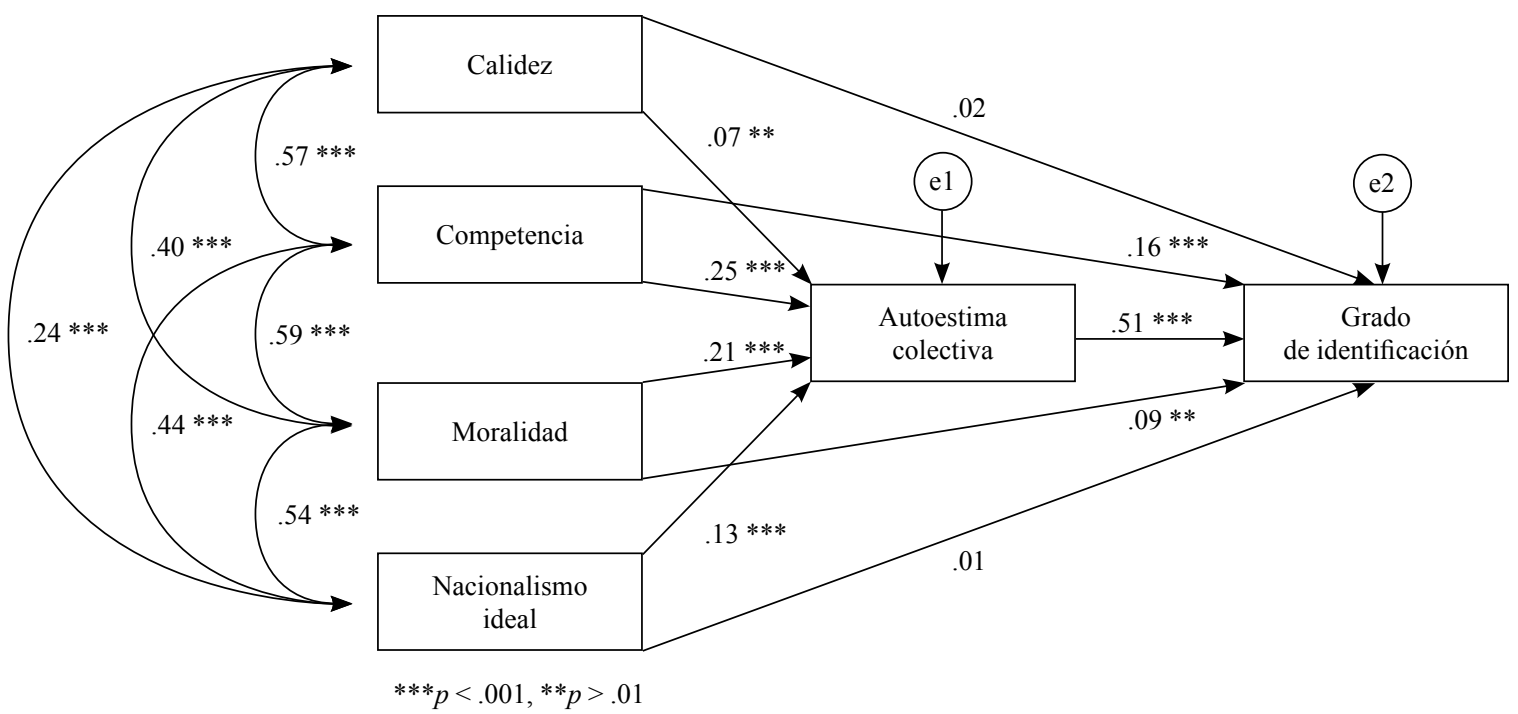

Figura 1. Análisis general de las influencias de las dimensiones autoestereotípicas en la autoestima colectiva y la identidad nacional (modelo hipotético)

autoestima colectiva en la identificación nacional. La figura 1 resume el modelo hipotético.

El modelo hipotético corresponde a un modelo completamente saturado y presenta niveles de ajuste excelentes: $G F I=1 ; C F I=1$. Sin embargo, los indicadores de error son ambivalentes: $R M R=.001$, $R M S E A=.374$. Adicionalmente, como se puede apreciar en los valores de beta estandarizados, las influencias de la calidez y el nacionalismo ideal en el grado de identificación no son significativas, $y$ la influencia de la calidez en la autoestima colectiva, aunque significativa, representa un tamaño del efecto muy pequeño.

En ese sentido, se propone un modelo alternativo que mantenga los niveles de ajuste obtenidos y que mejore los resultados de los indicadores de error. Conceptualmente, el nuevo modelo mantiene la idea general de que los autoestereotipos inciden en la valoración e identificación con el grupo nacional. Sin embargo, se eliminan las líneas de regresión de las dimensiones de calidez y nacionalismo ideal del grado de identificación, pues — como se ha podido apreciar - los efectos de estas dimensiones no son significativos. El modelo alternativo se presenta en la figura 2.
El modelo alternativo, eliminando las influencias de los autoestereotipos de calidez y nacionalismo ideal en el grado de identificación, presenta niveles de ajuste excelentes, $\chi^{2} / g l=.29 ; G F I=1$; $C F I=1$, y mejora los indicadores de error a niveles óptimos: $R M R=.003 ; R M S E A=.001$. En todos los casos, los valores de beta estandarizados son significativos y presentan tamaños del efecto moderados, con excepción de la influencia del autoestereotipo de calidez en la autoestima colectiva, que presenta un tamaño del efecto pequeño.

\section{Discusión}

Los resultados del estudio sugieren la presencia de cuatro dimensiones autoestereotípicas relevantes para la comprensión de las identificaciones nacionales en países de América Latina: calidez, competencia, moralidad y nacionalismo ideal. La estructura factorial exploratoria es validada de modo parcial por el análisis factorial confirmatorio; en tanto que los niveles de ajuste son solo aceptables y los resultados sobre los indicadores de error resultan ambivalentes. En ese sentido, es necesario seguir evaluando el funcionamiento del 


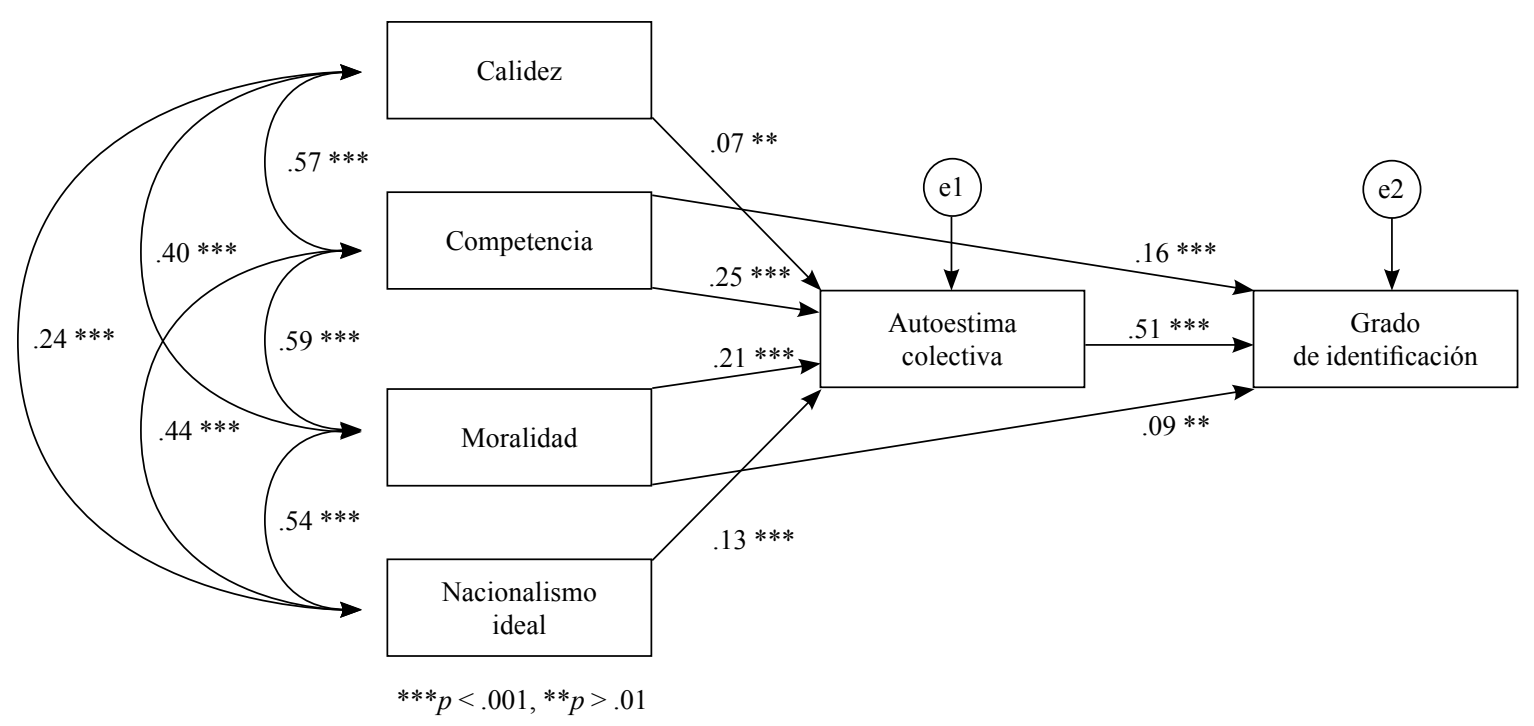

Figura 2. Análisis general de las influencias de las dimensiones autoestereotípicas en la autoestima colectiva y la identidad nacional (modelo alternativo)

cuestionario de autoestereotipos nacionales en muestras heterogéneas de distintos países de América Latina. Asimismo, una recomendación que deriva del presente estudio es analizar la necesidad de incorporar algunos adjetivos a la escala y hacer una revisión constante de las adaptaciones lingüísticas realizadas, sobre todo en aquellos adjetivos cuyo uso se relaciona a la jerga y expresiones idiosincráticas de cada país. No obstante, con todo lo referido, es importante destacar que los resultados del presente estudio son sumamente interesantes y permiten una aproximación apropiada al fenómeno de las representaciones identitarias nacionales en América Latina.

Sobre los contenidos estereotípicos propiamente dichos, las dimensiones de calidez y competencia tienen cierta correspondencia conceptual con la propuesta del modelo de contenidos mixtos de los estereotipos (Fiske et al., 2003; Fiske et al., 2002). De manera específica, el atributo de calidez es el predominante en la muestra general, y es el más consensual en 5 de los 6 países donde se desarrolló el estudio (en Chile es el segundo atributo más importante). La calidez como dimensión autoestereotípica aparece vinculada al colectivismo latinoame- ricano como síndrome cultural, caracterizado por relaciones interdependientes dentro del endogrupo, donde se promueve la evitación de conflictos, la comprensión, la empatía afectiva, la expresión abierta de afectos positivos, lo que constituye la base del mantenimiento de buenas relaciones interpersonales, y refuerza el escenario cultural del ser simpático con la intención de mantener la estabilidad y armonía del endogrupo (Gissi, Zubieta \& Páez, 2002; Kimble et al., 2002). Sin embargo, la dimensión de calidez incorpora un elemento adicional de viveza y adaptabilidad, expresadas en una idea de astucia que en ocasiones puede relacionarse con expresiones conductuales de una baja moralidad y un alto pragmatismo que no necesariamente son vistas de manera negativa por los miembros del endogrupo (cf. Beramendi, 2014; Monsegur et al., 2014), de lo cual resulta que este segundo aspecto es una variante de la descripción original de calidez propuesta en el modelo de contenidos mixtos, donde la calidez viene acompañada de algunos elementos de moralidad, como la sinceridad (Fiske et al., 2002), lo que no ocurre en este caso.

La competencia es el segundo atributo autoestereotípico más importante para la muestra general, 
y es consensualmente el segundo más importante en cinco de los seis países considerados en el estudio (en Chile este atributo ocupa el primer lugar). Sus contenidos están claramente relacionados con la descripción del modelo de contenidos mixtos (Fiske et al., 2002) y aluden a un conjunto de características y capacidades de los miembros del endogrupo para afrontar distintas situaciones de forma exitosa.

Hasta este punto, es correcta la aseveración realizada en un acápite previo sobre la utilidad del modelo de Fiske et al. (2002) para analizar no solo estereotipos, sino también autoestereotipos; al menos en el contexto de los países donde se efectuó el estudio. Sin embargo, aunque la aproximación descrita es correcta, resulta insuficiente tal como lo demuestra la presencia de dos dimensiones autoestereotípicas adicionales.

La moralidad aparece como una tercera dimensión autoestereotípica. Su conformación sugiere la importancia del aspecto moral en las representaciones estereotípicas del endogrupo nacional, así como en la autoestima colectiva y la identificación con este. Sin embargo, para la muestra en general, su presencia es neutral, es decir, no se percibe moralidad ni su opuesto de manera clara. La muestra brasileña es la que reporta un menor nivel de moralidad como contenido autoestereotípico.

La dimensión nacionalismo ideal es interesante, porque rompe con la noción del nacionalismo como ideología (Miles, 2000). En este escenario, la dimensión encontrada propone que el sentimiento nacional positivo debe venir acompañado de una visión cuestionadora y de desarrollo. Esta agrupación de adjetivos, lejos de ser espuria o azarosa, describiría al ciudadano en un sentido amplio, aquel que puede tener sentimientos positivos hacia el endogrupo, pero que cuestiona sus aspectos negativos y rompe con ideologías nacionalistas de naturaleza autoritaria o conservadora, las cuales, si bien garantizan cierta cohesión, identifican a los críticos al sistema como amenazantes o socialmente desviados (cf. Altemeyer, 2004). Este atributo parte de una asociación interesante de ideas, pero no se percibe como distintivo de ninguno de los endogrupos nacionales que participan en el estudio.

Los análisis de correlaciones sugieren que la expresión positiva de todas las dimensiones se relaciona a una mayor autoestima colectiva y a una mayor identificación nacional. Estas se reproducen en todos los países evaluados, con la excepción del caso brasileño, donde la calidez no está asociada con el nacionalismo ideal. A grandes rasgos, esas relaciones positivas entre las dimensiones autoestereotípicas, la autoestima colectiva y el grado de identificación sugieren lo propuesto por Smith et al. (2005) y Espinosa (2011), quienes encontraron que la autoestereotipia positiva tiene efectos positivos en la identificación con el endogrupo nacional. En el presente estudio, sobre este razonamiento se proponen los análisis de senderos y se aprecia que la calidez, la competencia, la moralidad y el nacionalismo ideal inciden positivamente en la autoestima colectiva y la autoestima colectiva; así mismo, la competencia y la moralidad inciden positivamente en la identificación con el endogrupo nacional.

Quizás el resultado más interesante y que llama la atención es que la calidez, siendo el atributo autoestereotípico que supuestamente mejor describiría a los endogrupos nacionales, se encuentre relacionado con la identificación con el país, mas no influye directamente en esta. Esto podría acontecer porque los autoestereotipos de calidez, si bien resultan positivos, no se vinculan necesariamente con grupos de alto estatus y con acceso a recursos (Fiske et al., 2002). De hecho, los estereotipos de calidez suelen estar relacionados con grupos de bajo estatus, percibidos como simpáticos pero incompetentes o como "buenos salvajes" (Espinosa, 2011). Entonces, la calidez sería una dimensión autoestereotípica determinada por las características culturales propias del colectivismo latinoamericano, que cumplen una función de representación positiva, casi hegemónica en los países latinoamericanos y que actúa como una suerte de "premio consuelo" identitario. Esto incide débilmente en la valoración positiva del 
endogrupo nacional, pero no incrementa la identificación con este, pues ser cálido no necesariamente asegura ser más moral o más competente, que sí son aspectos que parecen tener impacto en el estatus relativo de los grupos sociales.

Otro elemento que puede atentar contra la influencia de la calidez en la autoestima y el grado de identificación tiene que ver con la presencia de este elemento de pragmatismo y baja moralidad vinculado a la calidez, que aunque discursivamente es tolerado, y hasta considerado positivo en los países de la región, finalmente, supone un problema social que afecta la confianza interpersonal a niveles societales (e. g., Beramendi, 2014; Beramendi \& Zubieta, 2013; Monsegur et al., 2014). Por otra parte, el efecto positivo de la dimensión de competencia tanto en la autoestima colectiva como en el grado de identificación tiene que ver con la vinculación de estos atributos con el estatus y el acceso a recursos por las propias capacidades (Fiske et al., 2002; Fiske et al., 2003). La moralidad también ejerce efectos positivos tanto en la autoestima colectiva como en el grado de identificación y esto parece vincularse al hecho de que la percepción y expectativas positivas de los miembros del endogrupo producen más confianza interpersonal y más cohesión endogrupal (cf. Beramendi, 2014; Van Vugt $\&$ Hart, 2004). Finalmente, el nacionalismo ideal supone una aproximación crítica sobre la identificación nacional. En ese sentido, la representación de conciudadanos comprometidos con sus países, críticos con los aspectos negativos de las situaciones sociales en estos y que valoran positivamente el desarrollado, parece tener un efecto directo en la autoestima colectiva; pero no necesariamente incrementa el grado de identificación con el endogrupo nacional. Quizás porque esta dimensión se encuentra en un nivel de representación más desiderativo que concreto.

Los resultados del estudio han mostrado autoestereotipos con contenidos claros y consistentes que son compartidos por los participantes de los distintos países investigados. Sin embargo, a pesar de los aspectos en común, las diferencias específicas en las magnitudes de las relaciones de estos contenidos por país sugieren que las representaciones hegemónicas de estos autoestereotipos van a presentar diferencias en su interior. Para tal fin, el análisis de conglomerados permitió encontrar tres grandes segmentos de ciudadanos según sus puntuaciones en los autoestereotipos: los optimistas, los pesimistas y los ambivalentes. El análisis de la prevalencia de estos segmentos por país da resultados diferenciados. Así, la muestra colombiana es la única en que se aprecia un claro predominio del segmento optimista y en Chile se aprecia un claro predominio del segmento pesimista. En el resto de países, se aprecia esta tendencia a las representaciones identitarias ambivalentes como predominantes (cf. Espinosa, 2011), lo cual sugiere que la presencia de la identidad social negativa, aún continúa siendo un riesgo potencial en los ciudadanos de algunos países de la región (Espinosa, 2003; Monsegur et al., 2014; Montero, 1996a, 1996b). Finalmente, cabe resaltar que la distribución de estos segmentos no es estadísticamente representativa de la realidad de los países investigados, pero plantea preguntas relevantes sobre los procesos psicosociales y políticos a los que la población de clase media se está enfrentando, pues la definición de las identidades es un fenómeno psicológico contextualizado (cf. Beramendi, 2014; Beramendi \& Zubieta, 2013; Espinosa, 2011; Montero, 1996a, 1996b).

\section{Referencias}

Abrams, D. \& Hogg, M. A. (1988). Comments on the motivational status of self esteem in social identity and intergroup discrimination. European Journal of Social Psychology, 18, 317-334.

Altemeyer, B. (2004). The other "authoritarian personality”. En J. T. Jost y J. Sidanius (Eds.), Political psychology (pp. 85-107). New York: Psychology Press.

Banchs, M. A., Cadenas, J. M., Domínguez, D., \& Montero, M. (1993). Identidad nacional: 
permanencia y cambio. Revista Interamericana de Psicología, 27, 107-114.

Bar-Tal, D. (1996). Las creencias grupales como expresión de identidad social. En J. F. Morales, D. Páez, J. C. Deschamps, \& S. Worchel (Eds.), Identidad social: aproximaciones psicosociales a los grupos y a las relaciones entre grupos (pp. 255-286). Valencia: Promolibro.

Béjar, R. \& Cappello, H. M. (1986). La identidad y carácter nacionales en México: la frontera de Tamaulipas. Revista de Psicología Social, 1, 153-166.

Beramendi, M. (2014). Percepción del sistema normativo, transgresión y sus correlatos psicosociales en Argentina. Tesis doctoral no publicada, Facultad de Psicología, Universidad de Buenos Aires, Argentina.

Beramendi, M. \& Zubieta, E. (2013). Identidad nacional y relaciones interpersonales en una cultura donde la norma es la transgresión. Psicología Politica, 26, 165-177.

Carteri, K. K. (2008). A identidade nacional brasilei$\mathrm{ra}$. Recuperado de: http://biblioteconomiaepatrimonio.blogspot.com/2008/07/identidade-nacional-brasileira-karin.html

Cuddy, A. J., Fiske, S. T., \& Glick, P. (2007). The BIAS Map: Behaviors from Intergroup Affect and Stereotypes. Journal of Personality and Social Psychology, 92, 631-648.

D’Adamo, O. \& García Beaudoux, V. (1995). El argentino feo. Buenos Aires: Losada.

Espinosa, A. (2003). Identidad social e identidad nacional en una muestra de triciclistas de Juliaca. Tesis de licenciatura no publicada, Facultad de Letras y Ciencias Humanas, Especialidad de Psicología, PUCP.

Espinosa, A. (2011). Estudios sobre identidad nacional en el Perú y sus correlatos psicológicos, sociales y culturales. Tesis doctoral no publicada, Departamento de Psicología Social y Metodología de las CC. del Comportamiento, Universidad del País Vasco, España.
Espinosa, A. \& Beramendi, M. (2012). Diseño y evaluación de la Escala de Autoestereotipos Nacionales Argentinos. Manuscrito inédito.

Espinosa, A. \& Tapia, G. (2011). Identidad nacional como fuente de bienestar subjetivo y social. Boletín de Psicología, 102, 71-87.

Fiske, S. T., Cuddy, A. J., \& Glick, P. (2003). Emotions up and down: Intergroup emotions result from perceived status and competition. En D. M. Mackie \& E. R. Smith (Eds.), From prejudice to intergroup emotions: Differentiated reactions to social groups (pp. 247-264). Nueva York: Psychology Press.

Fiske, S. T., Cuddy, A. J., Glick, P., \& Xu, J. (2002). A model of (often mixed) stereotype content: Competence and warmth respectively follow from perceived status and competition. Journal of Personality and Social Psychology, 82, 878-902.

Gissi, J., Zubieta, E., \& Páez, D. (2002). Cultura y valores: la identidad social y cultural de América Latina. En F. J. Morales, D. Páez, D. Asún, \& A. L. Kornblit (Eds.), Psicología social (pp. 201-222). Buenos Aires: Pearson Education.

Hemphill, J. F. (2003). Interpreting the magnitudes of correlation coefficients. American Psychologist, 58, 78-80.

Herranz, K. \& Basabe, N. (1999). Identidad nacional, ideología política y memoria colectiva. Psicología Politica, 18, 31-47.

Hogg, M. A. \& Abrams, D. (1988). Social identifications: A social psychology of intergroup relations and group processes. London: Routledge.

Hogg, M. A. \& Ridgeway, C. (2003). Social identity: Sociological and social psychological perspectives. Social Psychology Quarterly, 66, 97-100.

Keillor, B., Tomas, G. \& Hult, M. (1999). A five-country study of national identity implications for international marketing research and practice. International Marketing Review, 16, 65-82.

Kimble, C., Hirt, E., Díaz-Loving, R., Hosch, H., Lucker, G. W., \& Zárate, M. (2002). Psicología 
social de las Américas. México D. F.: Pearson Prentice-Hall.

Lorenzi-Cioldi, F. \& Döise, W. (1990). Levels of analysis and social identity. En D. Abrams \& M. A. Hogg (Eds.), Social identity theory: Constructive and critical advances (pp. 71-88). New York: Harvester Wheatsheaf.

Luhtanen, R. \& Crocker, J. (1992). A collective self-esteem scale: Self evaluation of one's identity. Personality and Social Psychology Bulletin, 18, 302-318.

Lyons, A. C., Cude, B., Lawrence, F. C., \& Gutter, M. (2005). Conducting research online: Challenges facing researchers in family and consumer sciences. Family and Consumer Sciences Research Journal, 33, 341-356.

Marques, J., Páez, D., Valencia, J., \& Vincze, O. (2006). Effects of group membership on the transmission of negative historical events. Psicología Política, 32, 79-105.

Mezulis, A. H., Abramson, L. Y., Hyde, J. S., \& Hankin, B. L. (2004). Is there a universal positivity bias in attributions?: A meta-analytic review of individual, developmental and cultural differences in the self-serving attributional bias. Psychological Bulletin, 130, 711-747.

Miles, R. (2000). Nacionalismo. En E. Cashmore, E. (Ed.), Dicionário de relações étnicas e raciais (pp. 383-386). São Paulo: Selo Negro.

Miller, D. R. (1983). Self, symptom, and social control. En T. R. Sarbin \& K. E. Scheibe (Eds.), Studies in social identity (pp. 319-338). New York: Praeger.

Montero, M. (1992). Atracción y repulsión: identidad nacional en hijos de inmigrantes. Boletín de Psicología, 37, 21-42.

Montero, M. (1996a). Identidad social negativa y crisis socioeconómica: un estudio psicosocial. Revista Interamericana de Psicología, 30, 43-85.

Montero, M. (1996b). La identidad social negativa: un concepto en busca de teoría. En J. F. Morales, D. Páez, J. C. Deschamps \& S. Worchel (Eds.), Identidad social: aproximaciones psicosociales a los grupos y a las relaciones entre grupos (pp. 395-415). Valencia: Promolibro.

Monsegur, S., Espinosa, A., \& Beramendi, M. (2014). Identidad nacional y su relación con la dominancia social y la tolerancia a la transgresión en residentes de Buenos Aires (Argentina). Interdisciplinaria, 31(1), 5-23.

Morales, J. F. \& Páez, D. (1996). Estereotipos, discriminación y relaciones intergrupos en España y en Latinoamérica. En R. Y. Bourhis, J.-P. Leyens, J. F. Morales, \& D. Páez (Eds.), Estereotipos, discriminación y relaciones entre grupos (pp. 1-22). Madrid: McGraw-Hill.

Nigbur, D. \& Cinnirella, M. (2007). National identification, type and specificity of comparison and their effects on descriptions of national character. European Journal of Social Psychology, 37, 672-691.

Owens, T. J. (2006). Self and identity. En J. Delamater (Ed.), Handbook of social psychology (pp. 205-232). New York: Springer.

Páez, D., Marques, J., Valencia, J., \& Vincze, O. (2006). Dealing with collective shame and guilt. Psicología Política, 32, 59-78.

Pérez de León, P. (2007). Identidades, actitudes y estereotipos nacionales y supranacionales en una muestra uruguaya. Ciencias Psicológicas, 1, 81-102.

Pérez, T. (1999). Nación, identidad nacional y otros mitos nacionalistas. Oviedo: Nobel.

Richard, F. D., Bond Jr., C. F., \& Stokes-Zoota, J. J. (2003). One hundred years of social psychology quantitatively described. Review of General Psychology, 7, 331-363.

Roselli, N. (2000). Representación social de "los argentinos", "los españoles", "los latinoamericanos" y "los europeos" en estudiantes universitarios argentinos. Revista Latinoamericana de Psicología, 32, 127-160.

Rottenbacher, J. M. \& Espinosa, A. (2010). Identidad nacional y memoria histórica colectiva en el Perú. Un estudio exploratorio. Revista de Psicología, 28, 147-174. 
Salazar, J. M. (1996). Identidad social e identidad nacional. En J. F. Morales, D. Páez, J. C. Deschamps, \& S. Worchel (Eds.), Identidad social: aproximaciones psicosociales a los grupos y a las relaciones entre grupos (pp. 495-515). Valencia: Promolibro.

Salazar, J. M. \& Salazar, M. A. (1998). Estudios recientes acerca de identidades nacionales en América Latina. Psicología Política, 16, 75-93.

Salgado, C. (1999). ¿Quiénes somos los peruanos?: una perspectiva psicológica de la identidad nacional Lima: Escuela Profesional de Psicología de la Universidad San Martín de Porres.

Smith, P. B., Giannini, M., Helkama, K., Maczynski, J., \& Stumpf, S. (2005). Positive auto-stereotyping and Self-construal as predictors of national identification. International Review of Social Psychology, 18, 65-90.

Stangor, C. (2009). The study of stereotyping, prejudice, and discrimination within social psychology: A quick history of theory and research. En T. Nelson (Ed.), Handbook of prejudice, stereotyping and discrimination (pp. 1-22). New York: Psychology Press.

Fecha de recepción: 30 de julio de 2014 Fecha de aceptación: 9 de septiembre de 2015
Tajfel, H. (1982). Social psychology of intergroup relations. Annual Review of Psychology, 33, 1-39.

Tajfel, H. (1984). Grupos humanos y categorías sociales: estudios de psicología social. Barcelona: Herder.

Van Vugt, M. \& Hart, C.M. (2004). Social identity as social glue: The origins of group loyalty. Journal of Personality and Social Psychology, 86, 585-598.

Vignoles, V. L., Regalia,C., Manzi, C., Golledge, J., \& Scabini, E. (2006). Beyond self-esteem: Influence of multiple motives on identity construction. Journal of Personality and Social Psychology, 90, 308-333.

Vives, J. (1994). Identidad mexicana: un proceso con cinco siglos de duración. Psicología Iberoamericana, 2(3), 14-20.

Weber, J. A. \& Bradley, K. D. (2006). Strengths and weaknesses of conducting web-based surveys: A review of the literature. Paper presented at the Mid-Western Educational Research Association annual meeting, Columbus, $\mathrm{OH}$. Recuperado de http://www.uky.edu/ kdbrad2/WebbasedSurveys.pdf 
